\title{
Types de fusions-acquisitions et évolution de l'emploi des entreprises restructurées
}

Illustration à partir de données françaises d'entreprises (2000-2004)

Types of mergers and acquisitions and evolution of the employment of restructuring firms: descriptive results from the french database Citrus

(2000-2004)

\section{Matthieu Bunel, Richard Duhautois et Lucie Gonzalez}

\section{(2) OpenEdition}

\section{Journals}

Édition électronique

URL : http://journals.openedition.org/travailemploi/4123

DOI : $10.4000 /$ travailemploi.4123

ISSN : 1775-416X

Éditeur

DARES - Ministère du Travail

Édition imprimée

Date de publication : 30 mars 2009

Pagination : 53-65

ISSN : 0224-4365

Référence électronique

Matthieu Bunel, Richard Duhautois et Lucie Gonzalez, " Types de fusions-acquisitions et évolution de l'emploi des entreprises restructurées », Travail et Emploi [En ligne], 117 | janvier-mars 2009, mis en ligne le 30 mars 2011, consulté le 30 avril 2019. URL : http://journals.openedition.org/ travailemploi/4123; DOI : 10.4000/travailemploi.4123 


\title{
Types de fusions-acquisitions et évolution de l'emploi des entreprises restructurées: illustration à partir de données françaises d'entreprises (2000-2004)
}

\author{
Matthieu Bunel (*), Richard Duhautois (**), Lucie Gonzalez (***)
}

\begin{abstract}
La littérature économique théorique et empirique sur les conséquences des fusions-acquisitions sur l'emploi fait ressortir une très forte hétérogénéité des fusions-acquisitions. La contribution de cet article est essentiellement descriptive et s'appuie sur l'exploitation d'une base de données récemment constituée par l'Insee, Citrus, répertoriant une large part des opérations de fusions-acquisitions ayant eu lieu sur le territoire français entre 2000 et 2004. Cette base, qui mobilise des informations légales et statistiques, identifie près de 3000 opérations par an. Ces données permettent de décrire la répartition des fusions-acquisitions en fonction de quatre dimensions, déterminantes en termes de conséquences sur l'emploi: le type d'opération; la taille relative des entreprises bénéficiaires et cédantes; le caractère intra- ou intersectoriel de l'opération; l'appartenance à un groupe de sociétés des entreprises participant à la fusion-acquisition. Une mesure approchée de l'existence et de l'ordre de grandeur des transferts d'effectifs associés aux transferts d'actifs lors des fusionsacquisitions est également proposée.
\end{abstract}

Les fusions-acquisitions recouvrent un ensemble d'opérations qui ont un impact sur l'activité productive courante des entreprises (opérations de production et de formation brute de capital fixe) par le biais de transferts d'activité (incluant la cession, la location ou la mise à disposition, de tout ou partie des actifs productifs entre deux unités légales ou plus). Le terme de restructuration s'applique quant à lui aux fusions-acquisitions mais également aux réorganisations internes qui ont un impact négatif sur l'emploi. Comme le souligne Ravaran (2006) «plus la conséquence [des fusions-acquisitions] sur l'emploi est importante, plus le projet est appelé restructuration ». Par assimilation probablement, les fusions-acquisitions restent fréquemment perçues comme un phénomène essentiellement négatif: le plus souvent, ce sont les effets immédiats destructeurs d'emplois et dégradant les conditions de travail qui sont mis en exergue. Les effets de moyen terme, potentiellement positifs ou négatifs, autrement dit la dimension «créatrice» ou non du phénomène de « destruction créatrice» qu'est la fusion-acquisition,

* Université de technologie de Belfort Montbéliard: matthieu. bunel@utbm.fr

** Centre d'études de l'emploi: richard.duhautois@enpc.fr

*** Insee-Crest, Centre de recherche en économie et statistique: lucie.gonzalez@insee.fr

Ces travaux ont été initiés pour répondre à un appel d'offre émanant de la Mission Animation de la recherche de la DARES. sont assez souvent ignorés. En effet, si la recherche de gains de productivité peut se traduire à court terme par des réductions d'effectifs, la croissance générée à moyen terme, en cas de succès de l'opération de fusion-acquisition (croissance qui peut provenir par exemple d'un accroissement des parts de marché de l'entreprise lié à une meilleure compétitivité), peut conduire à une augmentation de l'emploi qui peut aller jusqu'à compenser les destructions initiales. En plus d'être trop souvent cantonnée aux effets de très court terme, la perception des conséquences des fusions-acquisitions sur l'emploi s'appuie essentiellement sur un petit nombre d'opérations, les plus spectaculaires car dramatiques en termes d'emploi (plans sociaux). Elle ne repose donc pas sur une vision d'ensemble, représentative de l'hétérogénéité des fusions-acquisitions. Et ce d'autant plus que le zoom est généralement mis sur une seule entreprise et pas sur l'ensemble des entreprises parties prenantes de la fusion-acquisition, cédante(s) et bénéficiaire(s).

En France, la plupart des études portant sur les fusions-acquisitions s'appuient sur des études de cas ou sur des opérations faisant intervenir des entreprises cotées en bourse: elles se concentrent le plus souvent sur les grands groupes et présentent l'inconvénient de ne pas être représentatives de l'ensemble des entreprises touchées par les fusions-acquisitions. 


\section{Encadré 1 \\ Les données utilisées: les bases Citrus et Ficus de l'Insee}

Deux bases de données d'entreprises ont été appariées: Citrus, pour les informations sur les fusionsacquisitions, et Ficus pour les données d'emploi et les informations comptables.

La base de données Citrus (coordination des informations et des traitements sur les restructurations d'unités statistiques, cf. BeAuvoIs, RIVIĖRE, BeAUdeAu, CorBeL) construite par l'Insee est quasi-exhaustive pour les entreprises cotées (dont les titres sont inscrits à la cote officielle ou à celle du second marché d'une bourse de valeurs) et leurs filiales qui doivent publier les avis de fusions, de scissions et de convocation aux assemblées générales extraordinaires (AGE) au Bulletin des annonces légales obligatoires (Balo). L'autre source d'informations de type légal est le Bulletin officiel des annonces civiles et commerciales (Bodacc) déposé au greffe des tribunaux de commerce.

Pour les autres entreprises, plusieurs enquêtes sont mobilisées, notamment l'enquête annuelle d'entreprise (EAE) portant sur près de 200000 entreprises, l'enquête trimestrielle sur les investissements dans l'industrie portant sur 4000 entreprises, l'enquête Produits, charges et actifs portant sur 8000 entreprises d'au moins vingt salariés et dont le secteur d'activité est l'industrie, le commerce ou les services. Enfin, d'autres fusions-acquisitions sont répertoriées lorsque des ruptures sont repérées par les statisticiens de l'Insee dans les immobilisations ou le chiffre d'affaires des entreprises.

Dans les données de Citrus, c'est le code MERE (mouvement élémentaire de restructuration de l'entreprise) qui donne l'information sur la fusion-acquisition du point de vue de l'entreprise: entreprise cédante ou bénéficiaire, caractéristique de l'apport (apport total, partiel...), type d'apport ou de contrat (apport strict, achat ou vente, contrat de location-gérance), changement éventuel de nature de l'entreprise (par exemple lorsqu'une entreprise cesse son activité industrielle ou commerciale pour devenir une holding), caractéristiques de l'entreprise partenaire...Étant donné l'hétérogénéité des sources, légales et statistiques, dont est issue la base Citrus, les données disponibles présentent des disparités. L'information contenue dans le code MERE, qui peut potentiellement être très détaillée, ne l'est en pratique dans nos données que dans $13 \%$ des opérations: dans $87 \%$ des cas, on peut seulement repérer l'entreprise bénéficiaire et l'entreprise cédante.

Dans la base Citrus, l'unité élémentaire retenue comme "fusion-acquisition" est un ensemble d'opérations intéressant un même ensemble d'entreprises, au titre d'une année donnée et à une date d'effet donnée. La date d'effet considérée ne correspond pas forcément à la date de prise de décision de l'opération dans le cadre de l'AGE: c'est une date d'effet fiscal et comptable rétroactif, qui coïncide le plus souvent avec la date de début de l'exercice comptable pendant lequel la fusion-acquisition a lieu, ce qui garantit une certaine cohérence temporelle avec les données comptables associées via la base de données Ficus.

La source Citrus est enrichie continuellement depuis sa création en 1998, avec notamment l'intégration de nouvelles sources d'informations. II faut donc interpréter l'augmentation du nombre d'opérations recensées avec précaution, car une partie s'explique par la qualité croissante de la base de données. C'est la raison pour laquelle les résultats sont le plus souvent présentés en moyenne sur la période 2000-2004 et les évolutions d'une année sur l'autre ne sont pas commentées en tant que telles. La période 2000-2004 a été retenue car elle apparaît relativement homogène en termes de qualité de la collecte des données et de couverture des entreprises.

La base Citrus ne contient pas d'informations sur les caractéristiques économiques des entreprises, c'est pourquoi cette base a été appariée avec la base Ficus (fichier complet unifié de Suse).

La base de données Ficus regroupe l'ensemble des entreprises imposées au titre des bénéfices industriels et commerciaux (BIC) et des bénéfices non commerciaux (BNC), hors micro-entreprises, et exclut les entreprises imposées aux bénéfices agricoles et les exploitations agricoles soumises aux BIC. Elle résulte de la confrontation de données administratives (liasses fiscales) et de données d'enquête (enquêtes annuelles d'entreprise).

D'un point de vue théorique, l'impact effectif global des fusions-acquisitions sur l'emploi est indéterminé, ne serait-ce que parce que les fusions-acquisitions forment un ensemble loin d'être homogène. Une littérature théorique abondante en finance et gestion d'entreprise ainsi qu'en économie industrielle fait apparaître des raisons très diverses à l'origine du développement des fusions-acquisitions (Coutinet, Sagot-Duvauroux, 2003). On peut regrouper ces analyses autour de six axes principaux: 1) la performance financière de l'entreprise; 2) la recherche de pouvoir de marché; 3) la gestion et le contrôle de l'équipe dirigeante; 4) l'utilisation de fonds excédentaires; 5) l'efficacité productive (économies d'échelle, d'envergure et diversification de l'activité); 6) la rupture de contrats implicites (voir Margolis (2006) et Bunel, Duhautois, Gonzalez (2008) pour une présentation plus détaillée de ces analyses). Le plus souvent, le motif de la fusionacquisition est difficile à repérer, et ce d'autant plus que plusieurs raisons peuvent être en jeu. En outre, l'impact effectif sur l'emploi reste très difficile à prévoir à partir du motif de la fusion-acquisition: en effet, en plus de la stratégie choisie à court terme et de ses répercussions directes sur l'emploi (décision de downsizing par exemple), il existe aussi un effet

- 54 - Travail et Emploi $n^{\circ}$ 117• Janvier-mars 2009 
indirect et plus tardif, plus incertain également, qui dépend du succès de la stratégie initialement retenue. Les fusions-acquisitions ne tiennent en effet pas toujours leurs promesses et se soldent fréquemment par un échec (SACHS-DuRAND, 2004).

C'est la raison pour laquelle nous nous appuierons surtout ici sur les études empiriques existantes sur le thème de l'impact des fusions-acquisitions sur l'emploi (évaluations ex post). Ces études font ressortir des caractéristiques des fusions-acquisitions déterminantes en termes de conséquences effectives sur l'emploi, et plus facilement observables que le motif réel de la fusion-acquisition. Il existe plusieurs travaux qui considèrent l'évolution conjointe de l'emploi des entreprises bénéficiaires et cédantes pour appréhender l'effet des fusionsacquisitions sur l'emploi: sur données américaines (notamment Brown et MEdofF, 1988), anglaises (Conyon, Girma, Thompson, Wright, 2002) et françaises (Bunel et al. (1), 2008). Gugler et Yurtoglu (2004) proposent une comparaison des effets des fusions-acquisitions aux Etats-Unis, en Europe continentale et au Royaume-Uni.

L'objectif de cet article est de compléter les analyses existantes en proposant un éclairage quantitatif plus représentatif de l'hétérogénéité des opérations de fusions-acquisitions: il s'articule autour des caractéristiques les plus déterminantes en termes d'effets observés sur l'emploi. Les données mobilisées proviennent de la base Citrus constituée par l'Insee (voir encadré 1). Cette base, constituée graduellement depuis 1998, mobilise des informations légales et statistiques. Elle répertorie un grand nombre d'opérations de fusions-acquisitions ayant eu lieu sur le territoire français. Un échantillon de près de 3000 fusions-acquisitions par an sur la période 2000 à 2004 est ainsi disponible.

Plus spécifiquement, cet article utilise ces données pour caractériser les opérations de fusionsacquisitions en précisant la répartition de ces opérations en fonction de quatre axes:

1) le type d'opération (absorption, cession partielle d'actifs, scission et changement de propriétaire) et l'ampleur du transfert d'effectifs qui accompagne le transfert d'actifs ;

2) la taille des entreprises et le rapport de «dominance» qui peut être mesuré en comparant la taille de l'entreprise bénéficiaire (acquéreuse) à celle de l'entreprise cédante (acquise);

3) le secteur d'activité et la dimension intrasectorielle (entreprises cédantes et bénéficiaires appartiennent au même secteur d'activité) ou intersectorielle (les entreprises appartiennent à des secteurs d'activité différents) de la fusion-acquisition;

(1) Les informations sur les fusions-acquisitions proviennent des fichiers MoDifications de STructure (MDST) pour la période 1993-1999.
4) l'appartenance à un groupe de sociétés des entreprises participant à la fusion-acquisition: on s'intéressera au cas où la fusion-acquisition fait entrer dans un groupe de sociétés une entreprise jusque-là indépendante, et notamment lorsque la tête de ce groupe est de nationalité étrangère.

En effet, dans les études empiriques, ces quatre types de caractérisation des fusions-acquisitions apparaissent déterminants dans l'évolution de l'emploi observée après une fusion-acquisition. Pour chacun de ces axes, on présentera les attendus théoriques et surtout les résultats des études empiriques concernant les effets de court et moyen terme sur l'emploi. Ils seront ensuite mis en perspective avec le poids de la caractéristique en question dans les fusions-acquisitions effectivement observées sur le territoire français au début des années 2000. Ces quatre aspects, passés en revue successivement dans cet article, ne sont bien évidemment pas séparés dans la réalité et peuvent se combiner. Préalablement à l'exposition de ces quatre aspects, nous préciserons les définitions économiques et statistiques des fusions-acquisitions retenues dans cet article et présenterons des statistiques descriptives globales s'appuyant sur ces définitions.

Dans l'ensemble de cet article, il faut garder à l'esprit que lorsqu'on parle des conséquences de fusions-acquisitions sur l'emploi, on ne se situe pas au niveau de l'économie française dans son ensemble, ni même au niveau d'un marché de biens ou services particulier, mais au niveau des entreprises participant aux fusions-acquisitions, qu'elles soient cédantes (acquises, totalement ou en partie) ou bénéficiaires (acquéreuses) de l'opération. Par exemple, il est possible que le bilan emploi d'une fusion ait pour conséquence un accroissement de l'emploi pour l'entreprise issue d'une fusion-acquisition, et que cette augmentation de l'emploi se fasse au détriment d'autres entreprises présentes sur ce marché (qui doivent alors réduire leurs effectifs ou disparaître), si bien que l'impact global peut se révéler négatif. Nous ne nous intéressons pas non plus à l'échec ou la réussite d'une fusion-acquisition en termes de faillite d'entreprise: étant donné les difficultés à appréhender la démographie des entreprises et les restructurations, il existe très peu d'études sur le sujet (BALDwIN, GORECKI, 1990).

\section{Qu'est-ce qu'une fusion- acquisition? Définitions économique et statistique}

Le terme «fusion-acquisition» vise :

1) les fusions-acquisitions au sens strict: fusions, scissions, apports partiels d'actifs ;

2) les opérations de vente (ou cession) ou achat (ou apport à titre onéreux) de fonds de commerce ou d'actifs productifs; 
3) les opérations de location-gérance, où la contrepartie des actifs circulants (stocks) ou incorporels (fonds de commerce) «prêtés» est constituée par des redevances de location.

Sont exclues de cette définition les opérations à caractère financier (y compris les apports en capital sans autre apport), les opérations de restructuration interne (transferts entre établissements ou réorganisation des structures de gestion) et les opérations de réorganisation interne de l'activité. La définition retenue exclut en particulier les cas où la prise de contrôle est une simple prise de participation (acquisition d'un nombre important de titres de la société cible qui peut aller jusqu'à la filialisation) sans transferts d'actifs physiques ni de salariés (2).

Le transfert d'actif peut avoir lieu sous trois formes: échanges de titres, transfert à titre onéreux (monétaire), ou apport mixte. L'acquisition peut être totale et viser l'ensemble de l'entreprise ou partielle et ne porter que sur une partie de l'entreprise (une branche d'activité, un ou plusieurs établissements...).

\section{Un phénomène rare, concentré sur un petit nombre d'entreprises}

La base de données Citrus mobilise des sources d'informations légales et d'enquêtes statistiques et permet d'identifier la plupart des fusions-acquisitions opérées sur le territoire français (encadré 1). Avec les conventions de définition des fusionsacquisitions issues de Citrus adoptées ici, on dénombre autour de 3000 opérations chaque année pour la période 2000-2004. Les fusions-acquisitions concernent un très petit nombre d'entreprises chaque année, autour de 5000 par an (bénéficiaires ou cédantes), ce qui représente autour de $0,2 \%$ du nombre total d'entreprises (tableau 1). Pour la majorité des entreprises, une fusion-acquisition reste un événement exceptionnel. BunEL et al. (2008) calculent que $89 \%$ des entreprises ayant participé à une fusion-acquisition sur le territoire français entre 1997 et 2001 ne l'ont fait qu'une fois sur la période, soit comme cédante, soit comme bénéficiaire.
Tableau 1 : Nombre d'opérations de fusions-acquisitions et d'entreprises bénéficiaires et cédantes dans les fichiers Citrus de l'Insee

\begin{tabular}{|l|l|l|l|l|l|}
\hline & $\mathbf{2 0 0 0}$ & $\mathbf{2 0 0 1}$ & $\mathbf{2 0 0 2}$ & $\mathbf{2 0 0 3}$ & $\mathbf{2 0 0 4}$ \\
\hline $\begin{array}{l}\text { Nombre d'opé- } \\
\text { rations de } \\
\text { fusion-acqui- } \\
\text { sition }\end{array}$ & 3043 & 3135 & 3410 & 3546 & 3339 \\
\hline $\begin{array}{l}\text { Nombre } \\
\text { d'entreprises }\end{array}$ & 4782 & 5089 & 5422 & 5660 & 5124 \\
\hline $\begin{array}{l}\text { Nombre } \\
\text { d'entreprises } \\
\text { bénéficiaires }\end{array}$ & 2138 & 2294 & 2365 & 2613 & 2334 \\
\hline $\begin{array}{l}\text { Nombre } \\
\text { d'entreprises } \\
\text { cédantes }\end{array}$ & 2786 & 2909 & 3183 & 3228 & 2942 \\
\hline
\end{tabular}

Source: Citrus 2000-2004.

Note: pour une année donnée, une même entreprise peut être à la fois cédante et bénéficiaire.

Ces entreprises rassemblent entre $10 \%$ et $12 \%$ des effectifs salariés selon les années (tableau 2). Il ne s'agit pas des effectifs «transférés» d'une entreprise à une autre lors de la fusion-acquisition, mais bien des effectifs de l'ensemble des entreprises parties prenantes des fusions-acquisitions.

Tableau 2: Poids économique des entreprises participant aux fusions-acquisitions

\begin{tabular}{|c|c|}
\hline & Moyenne 2000-2004 \\
\hline \multicolumn{2}{|l|}{ Nombre d'entreprises (en milliers) } \\
\hline $\operatorname{Total}^{(*)}$ & 2361 \\
\hline Fusion-acquisition $^{(* *)}$ & 5,2 \\
\hline Fusion-acquisition, en $\%$ du total & $0,2 \%$ \\
\hline \multicolumn{2}{|l|}{ Effectifs salariés (en millions) } \\
\hline Total $(*)$ & 15,1 \\
\hline Fusion-acquisition & 1,7 \\
\hline Fusion-acquisition, en $\%$ du total & $11 \%$ \\
\hline
\end{tabular}

Sources: Citrus 2000-2004; Ficus 2000-2004.

(*) Le total auquel il est fait référence correspond aux entreprises recensées dans le fichier Ficus ( $c f$. encadré 1$)$.

$(* *)$ «Fusion-acquisition» correspond aux entreprises (cédantes ou bénéficiaires) parties prenantes des fusions-acquisitions pour l'année considérée.

Les entreprises participant aux fusions-acquisitions sont en effet plus grandes en moyenne: leurs effectifs moyens sont de l'ordre de 50 fois ceux de l'ensemble des entreprises; mesurée par le montant des immobilisations corporelles(3), la taille moyenne des entreprises «fusions-acquisitions » est égale à 90 fois la taille moyenne de l'ensemble des entreprises.

(3) Immobilisations corporelles: terrains, constructions, installations techniques, matériel et outillages industriels, matériel de transport, de bureau et informatique, mobilier, avances et acomptes versés à des tiers pour l'acquisition d'immobilisations.
(2) Ces opérations de prise de participation financière s'effectuent via une offre publique d'achat (OPA) ou d'échange (OPE) pour les entreprises cotées ou par l'acquisition d'une partie du capital social pour les entreprises non cotées. 
Schéma 1 : Types de fusions-acquisitions

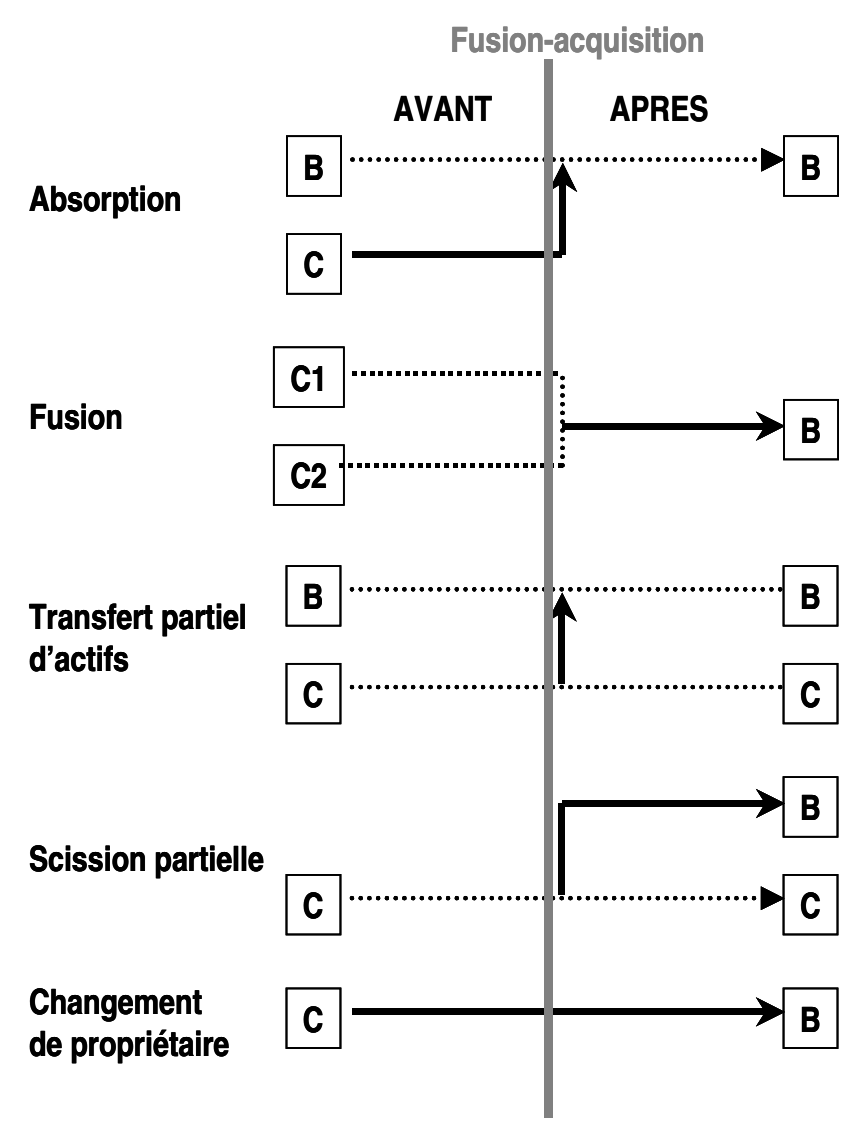

Note de lecture: B est l'entreprise bénéficiaire; C l'entreprise cédante. La flèche en trait gras représente le transfert d'actifs.

\section{Types de transferts d'actifs lors des fusions-acquisitions}

Le nom générique de fusion-acquisition recouvre des configurations multiformes. À partir des données dont nous disposons, quatre types sont repérables directement à l'aide du code MERE de la base Citrus (encadré 1), ou indirectement en fonction des mouvements observés sur les actifs, les effectifs et les codes identifiants, les entreprises (identifiants Sirene). Considérés du point de vue de la bénéficiaire $B$ ( $C$ étant la cédante), les types de transferts d'actifs observés dans les données sont les suivants (voir schéma 1):

- des absorptions : l'intégralité des actifs de l'entreprise C est absorbée par l'entreprise B; les fusions au sens strict (C1et $\mathrm{C} 2$ disparaissent pour devenir B) sont très peu nombreuses et de fait classées dans les absorptions;

- de simples changements de propriétaire : $\mathrm{C}$ devient $\mathrm{B}$;

- des transferts partiels d'actifs entre entreprises existantes: C se défait d'une partie de ses actifs au profit de B;

- des scissions partielles: C se scinde en plusieurs morceaux sans disparaître; un (ou plusieurs) de ces «morceaux» devient une entreprise nouvelle B (la scission serait totale si $\mathrm{C}$ disparaissait au profit de plusieurs entreprises nouvelles).

Dans la quasi-totalité des opérations $(99,4 \%$ sur la période 2000-2004), il n'y a qu'une seule entreprise cédante et une seule entreprise bénéficiaire. Dans la suite, les statistiques proposées reposent le plus souvent sur ces opérations «simples», ce qui facilite la lecture des résultats.

Chaque année, dans près de $80 \%$ des fusionsacquisitions, l'opération bénéficie à une entreprise qui existe déjà avant l'opération: il s'agit en effet, à parts égales, d'absorptions et de transferts partiels d'actifs (tableau 3). Dans un peu plus d'un cas sur deux, la cédante survit à l'opération (transferts partiels d'actifs et scissions partielles). On évalue alors le transfert médian d'actifs à environ $19 \%$ des immobilisations corporelles de l'entreprise cédante observées avant la fusion-acquisition(4).

(4) Un transfert médian de $19 \%$ des immobilisations corporelles signifie que pour la moitié des opérations le transfert est au plus égal à $19 \%$ des immobilisations corporelles et pour l'autre moitié, le poids du transfert est supérieur ou égal à $19 \%$. On présente les valeurs médianes car les valeurs moyennes sont trop sensibles aux valeurs extrêmes. 
Tableau 3 : Répartition des fusions-acquisitions par type d'opération

Total 2000 à 2004

\begin{tabular}{|l|r|}
\hline Absorptions & $(*)$ \\
\hline Transferts partiels d'actifs & $6366(39 \%)$ \\
\hline Scissions partielles & $6341(38 \%)$ \\
\hline Changements de propriétaires & $2613(16 \%)$ \\
\hline Non classé & $921(6 \%)$ \\
\hline Total & $232(1 \%)$ \\
\hline
\end{tabular}

Sources: Citrus 2000-2004; Ficus 2000-2004.

(*) Les fusions au sens strict (C1 et $\mathrm{C} 2$ disparaissent pour former $\mathrm{B}$ ), très marginales (huit pour l'ensemble de la période 2000-2004), sont comptabilisées avec les absorptions.

\section{Transferts d'actifs et transferts d'effectifs}

Pour chacun des types de fusion-acquisition construits, il est intéressant de savoir si le transfert d'actifs s'est accompagné ou non d'un transfert d'effectifs. Or, évaluer l'existence d'un transfert d'effectifs n'est pas aisé à partir des données dont nous disposons, c'est-à-dire à partir des stocks d'emploi pour chaque entreprise (cédante ou bénéficiaire) l'année de la fusion-acquisition et l'année précédant l'opération. L'idéal serait de disposer de l'information au niveau des salariés et de pouvoir les suivre d'une entreprise à une autre afin de calculer les flux de main-d'œuvre de façon exacte.

Nous nous contentons ici d'une méthode approchée et indirecte. En s'inspirant de la méthodologie développée par Brown et al. (1988)(5), on considère que le transfert d'effectifs entre cédante et bénéficiaire est "avéré» lorsque la variation de l'emploi de la bénéficiaire entre avant et après la fusion-acquisition représente au moins la moitié de la variation de l'emploi observée parallèlement chez la cédante. Sinon, on considérera que l'existence d'un flux de main-d'œuvre accompagnant le flux d'actifs est indéterminée. Ce seuil de la moitié est arbitraire. Il vise à tenir compte du fait que l'emploi des entreprises peut fluctuer pour d'autres raisons que l'éventuel transfert d'effectif: l'adaptation de la main-d'œuvre de l'entreprise à la conjoncture, plus ou moins favorable, les démissions ou fins de contrat à durée limitée $(6)$...

À partir des données Citrus, il ressort que le type de transfert d'actifs est lié à la présomption d'existence d'un transfert d'effectifs (tableau 4). Conformément à l'intuition, on trouve que les

(5) La méthodologie utilisée diffère cependant légèrement, ne serait-ce que parce que Brown et al. (1988) ne considèrent que des cas où la cédante disparaît totalement à la suite de la fusion-acquisition.

(6) À ces facteurs de variation de l'emploi, il faut ajouter les erreurs de mesure ou les valeurs manquantes pour l'emploi dans les données... mouvements d'actifs s'accompagnent de transferts d'effectifs dans la majorité des cas lorsqu'il s'agit d'un changement de propriétaire ( $87 \%$ en moyenne sur la période 2000-2004) ou d'absorption (77\%). Dans le cas des scissions partielles, le transfert d'effectif est «avéré» une fois sur deux. En revanche, il apparaît que les transferts partiels d'actifs ne se traduisent pas par un transfert simultané d'effectifs dans près de $80 \%$ des cas (7).

Les travaux empiriques soulignent que les opérations de fusions-acquisitions qui se caractérisent simultanément par un transfert d'actifs et d'effectifs ont des effets à moyen terme plus favorables à l'emploi (Brown et al., 1988; BUNEL et al., 2008). BROWN et al. (1988) sur données américaines montrent que les conséquences d'une fusion-acquisition sur l'évolution de l'emploi total des entreprises cédante et bénéficiaire est positif si le transfert d'actifs est accompagné d'un transfert d'effectifs: cet effet est estimé à $+3 \%$ trois ans après la fusionacquisition pour les absorptions (mergers) et à + $9 \%$ pour les changements de propriétaires (simple sales). Il est négatif sinon, et estimé à $-5 \%$ au bout de trois ans pour les assets only (transferts d'actifs), sans transfert notable d'effectifs. Sur données françaises pour la fin des années 1990, Bunel et al. (2008) trouvent des résultats convergents, avec un «effet emploi» au bout d'un an de $+4 \%$ en cas de transferts d'effectifs et de $-12 \%$ sinon.

Ainsi au vu de ces résultats empiriques et de ceux obtenus à l'aide de la base Citrus, on s'attend à ce que les conséquences sur l'emploi soient plus favorables dans le cas des changements de propriétaires et des absorptions, et plus souvent négatives lors des transferts partiels d'actifs. Sur un champ restreint à 11430 opérations de fusions-acquisitions pour 2000-2004 (sur un total de 16241 dans nos données), pour des raisons de faisabilité et de pertinence des calculs, on peut estimer les effectifs totaux transférés entre 1 et 2 millions de salariés pour 2000-2004, selon la méthode d'approximation retenue (méthodes (1), (2) ou (3), voir tableau 4).

\section{Tailles relatives des entreprises bénéficiaires et cédantes: un rapport de dominance?}

Les entreprises prenant part aux fusions-acquisitions sont de plus grande taille que les autres (tableau 2). Au sein de ces entreprises, les bénéfi-

(7) Ces relations entre type de transfert d'actifs et fréquence des transferts d'effectifs "avérés» sont confirmées par une analyse «toutes choses égales par ailleurs» (modélisation Logit), où on contrôle de la taille de départ des entreprises et du rapport de taille, du secteur d'activité et de l'appartenance à un groupe de sociétés de la bénéficiaire et de la cédante et du caractère inter-ou intrasectoriel de la fusion-acquisition. 
Tableau 4 : Repérage des transferts d'effectifs associés aux transferts d'actifs lors des fusions-acquisitions

\begin{tabular}{|c|c|c|c|c|c|c|c|c|}
\hline & \multirow{3}{*}{$\begin{array}{c}\text { Total }{ }^{(*)} \\
\text { 2000-2004 }\end{array}$} & \multicolumn{7}{|c|}{ Transferts d'effectifs «avérés» } \\
\hline & & \multirow{2}{*}{$\begin{array}{l}\text { Nombre d'opérations } \\
\left.\text { en \% du total }{ }^{*}\right)\end{array}$} & \multicolumn{3}{|c|}{$\begin{array}{l}\text { Effectif transféré } \\
\text { médian }(* *)\end{array}$} & \multicolumn{3}{|c|}{$\begin{array}{l}\text { Effectifs totaux transférés } \\
\text { (en milliers) }\end{array}$} \\
\hline & & & $(1)$ & $(2)$ & (3) & $(1)$ & $(2)$ & $(3)$ \\
\hline Changements de propriétaires & 740 & $642(87 \%)$ & 59 & 74 & 123 & 196 & 383 & 485 \\
\hline Absorptions & 4281 & $3227(77 \%)$ & 42 & 59 & 92 & 511 & 762 & 1029 \\
\hline Scissions partielles & 1850 & $892(48 \%)$ & 38 & 31 & 56 & 105 & 89 & 149 \\
\hline Transferts partiels d'actifs & 4559 & $999(22 \%)$ & 25 & 39 & 64 & 155 & 204 & 288 \\
\hline Ensemble & 11430 & $5810(51 \%)$ & & & & 968 & 1439 & 1952 \\
\hline
\end{tabular}

Sources: Citrus 2000-2004; Ficus 2000-2004.

(*) Pour que le seuil de $50 \%$ des variations d'effectif ait un sens, le champ est restreint aux fusions-acquisitions où la cédante compte au moins 6 salariés l'année avant la fusion-acquisition. En effet dans les autres cas, le calcul des pourcentages est très sensible à de faibles variations de l'emploi.

(**) Un transfert d'effectif médian de 59 salariés signifie que pour la moitié des opérations, le transfert est au plus égal à 59 salariés et pour l'autre moitié, le transfert est au moins égal à 59. On présente les valeurs médianes car les valeurs moyennes sont trop sensibles aux valeurs extrêmes.

Les effectifs transférés sont calculés selon trois méthodes:

(1) comme le minimum entre la variation d'effectifs de la cédante $(\mathrm{Tc})$ et celle de la bénéficiaire $(\mathrm{Tb}): \min (\mathrm{Tc}, \mathrm{Tb})$;

(2) comme la moitié de la variation d'effectifs de la cédante $(\mathrm{Tc}): 0,5 * \mathrm{Tc}$;

(3) comme la moyenne entre la variation d'effectifs de la cédante (Tc) et celle de la bénéficiaire $(\mathrm{Tb}):(\mathrm{Tc}+\mathrm{Tb}) / 2$.

ciaires apparaissent nettement plus grandes que les cédantes : sur la période 2000-2004, la taille médiane des bénéficiaires un an avant l'opération est environ le triple de celle des cédantes en cas d'absorption et de transfert partiel d'actifs (la taille de la bénéficiaire avant l'opération n'a pas de sens en cas de scission partielle et de changement de propriétaire, car la bénéficiaire se crée à l'occasion de la fusionacquisition) (tableau 5). Dans plus de $40 \%$ des cas cependant, la bénéficiaire est «plus petite» $(8)$ que la cédante (tableau 6). Non seulement la différence de taille entre la cédante et la bénéficiaire n'est pas toujours la règle, mais elle peut même s'observer dans le sens inverse de celui attendu.

Tableau 5 : Taille médiane des entreprises cédantes et bénéficiaires l'année précédant la fusion-acquisition selon le type d'opération

\begin{tabular}{|l|c|c|}
\hline & \multicolumn{2}{|c|}{$\begin{array}{c}\text { Taille médiane l'année } \\
\text { précédant } \\
\text { la fusion-acquisition }\end{array}$} \\
\hline & Cédante & Bénéficiaire \\
\hline Absorptions & 30 & 101 \\
\hline Transferts partiels d'actifs & 23 & 63 \\
\hline Scissions partielles & 87 & $-(*)$ \\
\hline Changement de propriétaire & 66 & $-(*)$ \\
\hline
\end{tabular}

Sources : Citrus 2000-2004; Ficus 2000-2004

La taille est mesurée par les effectifs salariés.

(*) La taille de la bénéficiaire avant l'opération n'a pas de sens en cas de scission partielle et de changement de propriétaire, car la bénéficiaire se crée à l'occasion de la fusion-acquisition.

(8) Bénéficiaire «plus petite» que la cédante signifie, avec les conventions utilisées dans le tableau 6 , que les effectifs de l'entreprise bénéficiaire sont inférieurs ou égaux à $75 \%$ des effectifs de la cédante.
Au-delà du simple aspect descriptif, le rapport de taille refléterait aussi un rapport de force et aurait une influence sur l'intégration au sein de la nouvelle entité des salariés cédés par l'entreprise acquise et, à moyen terme, sur la réussite de la fusion-acquisition. Sur données françaises appariées entreprisessalariés, MARGOLIS (2006) montre que les salariés issus de l'entreprise cédante ont une probabilité plus faible de rester dans la nouvelle entité que ceux issus de l'entreprise acquéreuse(9). En effet, si l'entreprise $\mathrm{B}$ (bénéficiaire) achète l'entreprise $\mathrm{C}$ (cédante) pour augmenter ses capacités de production et faire des économies d'échelle, il est fort probable que B utilise sa propre technologie plutôt que celle de C. De fait les salariés de B seront mieux adaptés (en termes de qualifications, de capital humain spécifique) que ceux issus de $\mathrm{C}$. Si B achète $\mathrm{C}$ pour améliorer sa gouvernance en essayant par exemple de réduire les rentes des salariés nées de contrats «implicites» conclus entre les salariés et l'entreprise $\mathrm{C}$, dans ce cas de figure également il est fort probable qu'après la fusion-acquisition les salariés provenant de l'entreprise cédante connaissent un sort moins favorable que ceux de l'entreprise bénéficiaire. Enfin, on ne peut exclure un certain «favoritisme» de la part de la direction de l'entreprise, privilégiant ses propres salariés au détriment des nouveaux venus. Cependant, MARGolis (2006) montre que les individus qui quittent l'entreprise après la fusion-acquisition (il n'est pas possible de savoir si leur départ est volontaire ou non) sont les plus «employables», c'est-à-dire ceux qui possèdent des caractéristiques associées à l'obtention rapide d'un nouvel emploi après un licenciement.

(9) Les effets sur la probabilité de départ des salariés issus des entreprises cédantes et bénéficiaires sont observés à court terme: ils disparaissent au bout de trois ans. 
Tableau 6 : Dominance - répartition du rapport de taille entre bénéficiaire et cédante : le déséquilibre est la règle

\begin{tabular}{lllllll}
\hline & $\begin{array}{c}\text { La bénéficiaire est plus petite que } \\
\text { la cédante }\end{array}$ & $\begin{array}{c}\text { Tailles } \\
\text { comparables }\end{array}$ & $\begin{array}{c}\text { La bénéficiaire est plus } \\
\text { grande que la cédante }\end{array}$ \\
& Moins de 0,33 & Entre 0,33 et 0,75 & Entre 0,75 et 1,25 & Entre 1,25 et 5 & Plus de 5 & Total \\
\hline Absorptions & $36 \%(33 \%)$ & $7 \%(6 \%)$ & $8 \%(5 \%)$ & $22 \%(18 \%)$ & $27 \%(38 \%)$ & $100 \%(100 \%)$ \\
Transferts partiels d'actifs & $36 \%(34 \%)$ & $8 \%(7 \%)$ & $7 \%(5 \%)$ & $19 \%(15 \%)$ & $30 \%(39 \%)$ & $100 \%(100 \%)$ \\
\hline
\end{tabular}

Sources: Citrus 2000-2004; Ficus 2000-2004.

La taille est mesurée par les effectifs (et par les immobilisations corporelles entre parenthèses) un an avant la fusion-acquisition.

Note de lecture: «moins de 0,33 » signifie que la taille de l'entreprise bénéficiaire est inférieure à $33 \%$ de la taille de la cédante. Pour les absorptions comme pour les transferts d'actifs, dans $36 \%$ des cas, la taille de la bénéficiaire est inférieure à $33 \%$ de la taille de la cédante.

Smeets, Ierulli, GibBs (2008), montrent même, en s'appuyant sur des données similaires pour le Danemark, que plus l'entreprise bénéficiaire «domine» l'entreprise cédante en termes de taille (mesurée par les effectifs salariés), meilleur est le sort, dans la nouvelle organisation, des salariés (de la bénéficiaire) déjà en place comparé aux salariés qui viennent de l'entreprise cédante(10). L'interprétation qui est faite de ce résultat repose sur la dimension organisationnelle des fusions-acquisitions et s'inspire de la littérature sur les conflits ethniques et l'assimilation. La culture d'une organisation comme l'entreprise peut en effet être définie comme des valeurs, des traditions, des normes, des croyances et des modèles de comportement. En résultent des règles formelles sur les valeurs et les aspirations de l'entreprise ainsi que des règles informelles et des réseaux. Des incompatibilités «culturelles » peuvent apparaître à plusieurs niveaux lorsque deux entreprises fusionnent, notamment dans les styles de management et les systèmes d'organisation comme les systèmes d'évaluation et de récompense. Ces différences culturelles peuvent être plus fortes lorsque les salariés sont de nationalités différentes. SMEETs et al. (2008) montrent que dans les entreprises bénéficiaires des fusions-acquisitions des années 1980 et 1990, la rotation de la main-d'œuvre (nombre de départs de salariés) est plus marquée pour les salariés qui proviennent de l'entreprise cédante que pour les salariés en place et qu'elle s'accompagne de l'embauche de nouveaux salariés, ce qui contredit l'idée selon laquelle les réductions d'effectifs après la fusion-acquisition seraient uniquement liées à des économies d'échelle. L'intégration des salariés issus du transfert constituerait ainsi un défi majeur pour l'entreprise acquéreuse et le succès de la fusion-acquisition, du fait

(10) Lorsque c'est l'entreprise cédante qui «domine» en taille l'entreprise bénéficiaire, elle connaît alors également une augmentation dans la rotation de sa main-d'œuvre. de la confrontation de deux cultures d'entreprises, et apparaîtrait de fait moins aisée que celle de nouvelles recrues. D'après SMEETs et al. (2008) les effets négatifs de la confrontation des deux cultures d'entreprise l'emporteraient sur les effets positifs (apparition de synergies, gain en créativité).

Dans les données françaises de fusions-acquisitions, on observe un déséquilibre dans la taille des deux entreprises dans plus de neuf cas sur dix. En effet, pour les absorptions et les transferts partiels d'actifs (11), les entreprises sont de taille comparable (rapport des effectifs initiaux entre $75 \%$ et $125 \%$ ) dans seulement $7 \%$ et $8 \%$ des cas respectivement $(5 \%$ si on mesure la taille par les immobilisations corporelles) (tableau 6).

\section{Aspects sectoriels: économies d'échelle et rationalisation de la production}

Sur la période 2000-2004, cinq secteurs d'activité sur treize regroupent $72 \%$ des entreprises parties prenantes des fusions-acquisitions (bénéficiaires ou cédantes): dans l'ordre d'importance décroissante, le commerce, les services aux entreprises, les biens intermédiaires, l'immobilier et les biens d'équipement (graphique 1).

Quel que soit le secteur considéré, le nombre de cédantes est relativement proche de celui des bénéficiaires, sauf dans l'immobilier qui compte deux fois plus d'entreprises acquises que d'entreprises acquéreuses. De fait, les fusions-acquisitions ne conduisent pas à une ré-allocation sectorielle marquée de l'activité des entreprises. 
Graphique 1 : Activités principales (APE) des entreprises cédantes et des bénéficiaires des fusions-acquisitions (2000-2004)

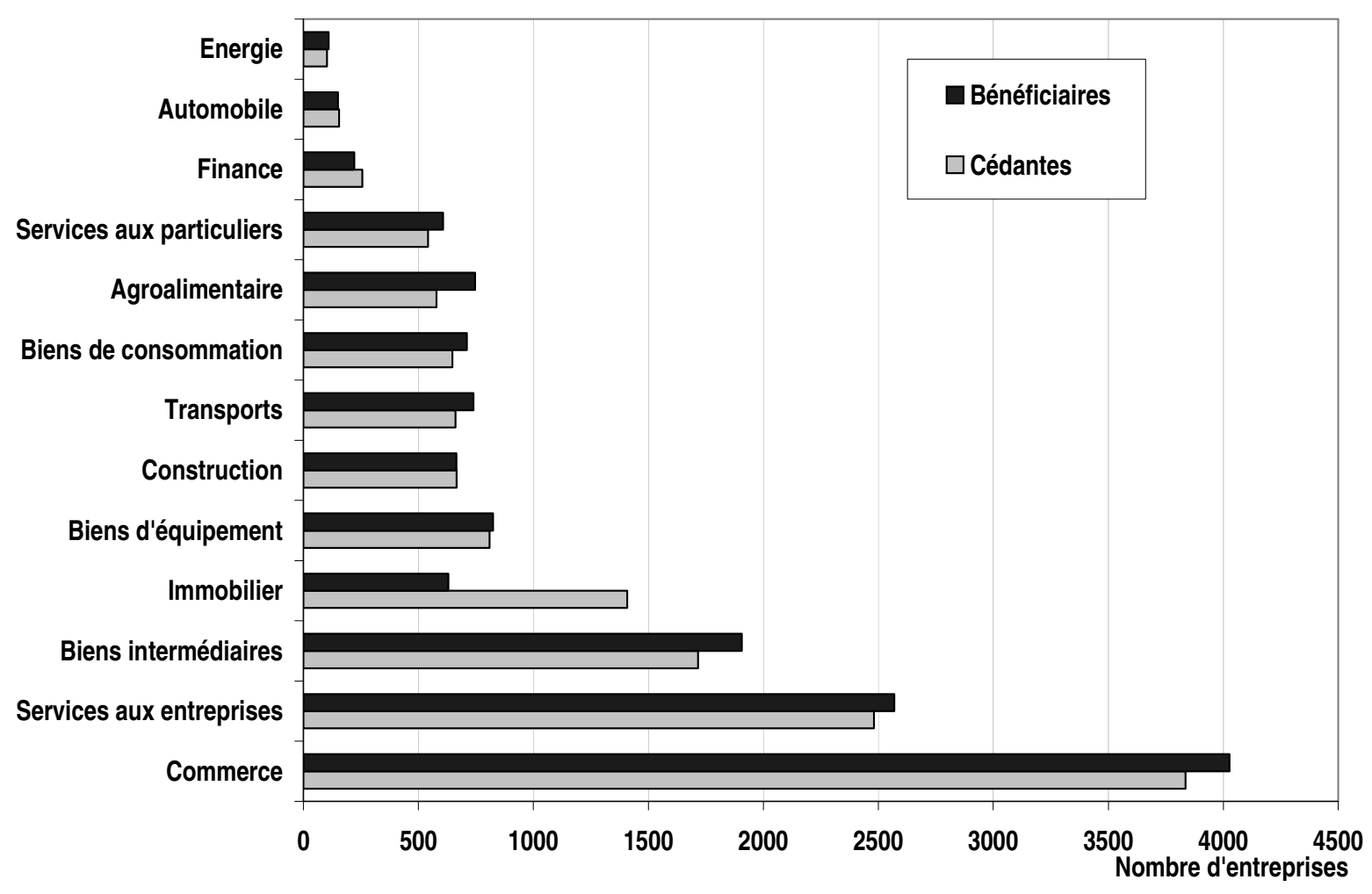

Sources: Citrus 2000-2004; Ficus 2000-2004.

Note de lecture: on a écarté les cas où l'une des entreprises est une holding (APE 741J) et les secteurs de l'agriculture, de l'éducation, santé, action sociale et de l'administration.

Tableau 7 : Fusions-acquisitions inter et intrasectorielles

\begin{tabular}{l|r|r|r|r|r}
\hline & $\begin{array}{r}\text { Changements } \\
\text { de propriétaire }\end{array}$ & Absorptions & $\begin{array}{c}\text { Transferts partiels } \\
\text { d'actifs }\end{array}$ & Scissions partielles & ENSEMBLE \\
\hline Inter-sectorielle & $17 \%$ & $28 \%$ & $31 \%$ & $37 \%$ & $31 \%$ \\
Intra-sectorielle & $2 \%$ & $5 \%$ & $5 \%$ & $6 \%$ & $5 \%$ \\
$\quad$ Activités assez proches & $81 \%$ & $67 \%$ & $64 \%$ & $57 \%$ & $64 \%$ \\
$\quad$ Activités très proches & & & \\
\hline
\end{tabular}

Sources: Citrus 2000-2004; Ficus 2000-2004.

Activités très proches $=$ un an avant la fusion-acquisition, cédante et bénéficiaire ont la même activité principale de l'entreprise (APE) au niveau le plus désagrégé (700 postes) de la Nomenclature d'activités française (NAF).

Activités assez proches = un an avant la fusion-acquisition, cédante et bénéficiaire ont la même APE au niveau plus agrégé (36 postes) de la nomenclature mais différent au niveau détaillé de la NAF en 700 postes.

Note 1: on a écarté les cas où l'une des entreprises est une holding (APE 741J).

Note 2: une entreprise peut exercer plusieurs types d'activité, par exemple une activité de production P et une activité commerciale C. C'est l'entreprise qui choisit son activité principale et donc le secteur dans lequel sera classé l'ensemble de ses activités. Lors d'un changement de propriétaire, l'acquéreur peut décider de privilégier une activité différente de celle choisie par son prédécesseur. De même, dans le cas d'une scission partielle ou d'un transfert partiel d'actifs, ce n'est pas forcément l'activité principale dont "hérite» la bénéficiaire (on peut même penser que ce serait plutôt l'inverse si

l'entreprise se recentre sur son cœur de métier). 
Une fusion-acquisition peut répondre à des logiques économiques ou stratégiques différentes. Si l'objectif est la concentration verticale, il s'agit de racheter des entreprises fournisseurs ou clientes, afin de maîtriser l'ensemble d'une filière de production et de distribution et de réduire les coûts de transaction notamment. Se concentrer horizontalement consiste à racheter des concurrents sur le même marché (dans la limite du respect d'un niveau suffisant de concurrence sur ce marché), ce qui permet à la fois de conforter son pouvoir de marché et aussi de faire des économies d'échelle. Une dernière logique, financière, est l'approche conglomérale: la diversification des activités correspond à une diversification de portefeuille et a un rôle essentiellement assurantiel (mutualisation des risques). L'intégration horizontale se traduit par l'acquisition d'entreprises ou de branches d'entreprises qui appartiennent à un secteur d'activité proche (fusionacquisition intrasectorielle), alors que l'intégration verticale et la constitution d'un conglomérat passent par l'achat d'entreprises qui exercent des activités différentes (fusion-acquisition intersectorielle). D'après le tableau 7 , un peu moins d'un tiers des fusions-acquisitions observées sur le territoire français entre 2000 et 2004 sont intersectorielles.

D'un point de vue théorique, lorsque les fusionsacquisitions ont lieu entre des entreprises qui n'appartiennent pas au même secteur d'activité (opération intersectorielle), l'effet sur l'emploi risque d'être moins négatif que lorsqu'elles s'opèrent au sein d'un même secteur (opérations «horizontales »), où les économies d'échelle dégagées et l'élimination des doublons et des surplus de capacité risquent de conduire à des réductions d'effectifs plus massives(12). Cet effet négatif s'atténuerait rapidement, deux trois ans après la fusion-acquisition (CONYON et al. 2002; Bunel et al. 2008). Toutefois, ce résultat peut être nuancé, si la fusionacquisition intersectorielle vise à sanctionner une mauvaise gestion de l'entreprise (rationalisation de la production).

Si on s'intéresse aux flux de salariés, SMEETs et al. (2008) montrent que si les entreprises viennent de secteurs d'activité différents (fusions-acquisitions intersectorielles), le turnover des salariés issus de la cédante est encore plus important, du fait des difficultés d'acculturation des nouveaux salariés. Il semblerait donc qu'en cas de fusion intrasectorielle, potentiellement génératrice de doublons et donc de réductions d'effectifs au niveau global, les salariés provenant de l'entreprise acquise soient moins défa-

(12) Il ressort d'une analyse « toutes choses égales par ailleurs» (modélisation Logit) des caractéristiques qui influencent les transferts d'effectifs «avérés» que le caractère intrasectoriel de la fusion-acquisition conduit à des transferts d'effectifs «avérés» plus fréquents que dans le cas d'une opération intersectorielle, ce qui est cohérent avec l'apparition de surplus de capacité et de doublons. vorisés par rapport aux salariés de la bénéficiaire puisqu'ils ont moins de risques de quitter l'entreprise que dans le cas d'une opération intersectorielle. Margolis (2006) présente des résultats similaires pour la France des années 1990: lorsque la transaction est intrasectorielle, on note peu de différences entre la probabilité de rester en emploi des salariés issus de la bénéficiaire et celle des salariés issus de l'entreprise acquise alors que les salariés de la cédante sont nettement moins bien lotis en cas de fusion-acquisition intersectorielle.

\section{L'appartenance à un «groupe de sociétés": la moitié des transactions sont intragroupes}

Si on considère la dimension groupe, il apparaît que les entreprises prenant part aux fusions-acquisitions appartiennent majoritairement à des groupes d'entreprises, qui se caractérisent par des liens de contrôle financier entre sociétés. En moyenne sur la période 2000-2004, les fusions-acquisitions ont lieu entre deux entreprises indépendantes dans seulement $13 \%$ des cas, ce qui est cohérent avec le poids croissant des entreprises des groupes dans l'économie française. Il y a plus de cédantes indépendantes que de bénéficiaires indépendantes et moins de cédantes que de bénéficiaires appartenant à un groupe privé, français ou étranger (graphique 2). De fait, les fusions-acquisitions sont l'occasion de ré-allocations des entreprises indépendantes vers les entreprises des groupes. Dans $23 \%$ des opérations observées sur la période 2000-2004, la fusionacquisition fait entrer une entreprise indépendante dans un groupe (13). Ce phénomène de ré-allocation est net en cas d'absorption et de transfert d'actifs.

À partir de données françaises sur la vague de fusions-acquisitions de la fin des années 1990, BunEL et al. (2008) montrent que les fusions-acquisitions impliquant des entreprises appartenant à un groupe ont des conséquences moins favorables sur l'emploi que celles ayant lieu entre entreprises indépendantes. L'effet est un peu moins négatif pour les opérations intragroupes, où cédantes et bénéficiaires appartiennent au même groupe. Or, parmi les fusions-acquisitions entre entreprises de groupes, la majorité (autour de $85 \%$ ) a lieu au sein d'un même groupe, soit environ la moitié du nombre total d'opérations de fusions-acquisitions.

Les prises de contrôle étranger (acquisitions d'entreprises françaises par un groupe étranger) représentent une part assez faible des fusions-acqui-

(13) Au sein de ces entrées dans les groupes, les «nationalisations ", où une entreprises entre dans un groupe public français, sont très rares : moins de $1 \%$ des fusions-acquisitions observées entre 2000 et 2004. 
Graphique 2: Appartenance à un groupe de sociétés des entreprises cédantes et bénéficiaires un an avant la fusion-acquisition

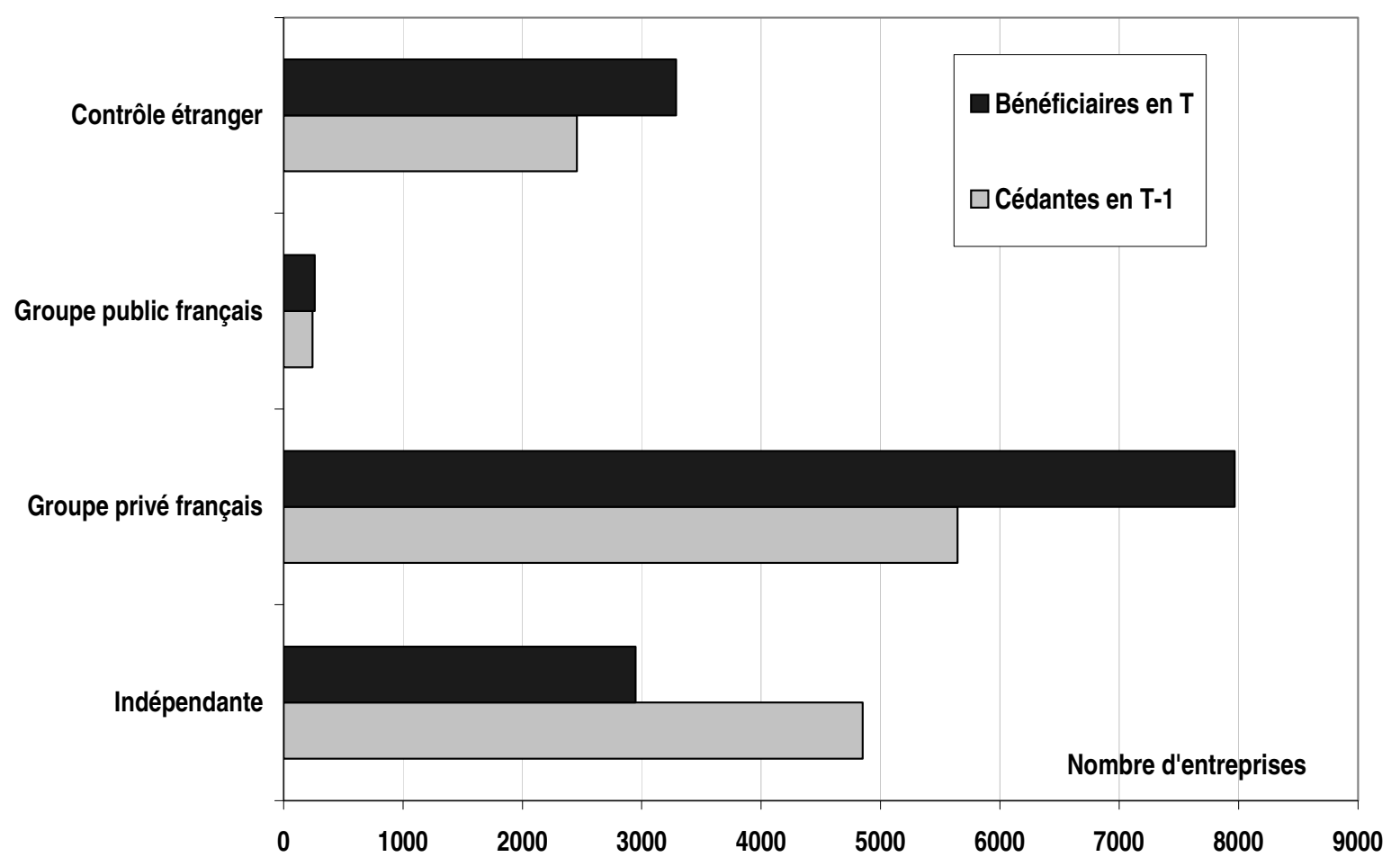

Sources : Citrus 2000-2004; Ficus 2000-2004.

sitions : 9\% en moyenne sur la période 2000-2004. Les développements théoriques sur ce thème sont à chercher dans la littérature sur les firmes multinationales dont il ressort deux types d'effets sur l'ajustement de l'emploi de sens opposés dont la résultante est indéterminée: d'une part, les multinationales procéderaient à des ajustements de l'emploi plus rapides et de plus grande ampleur que les firmes nationales; d'autre part, le niveau de qualification plus élevé des salariés des multinationales impliquerait une plus grande rigidité de la demande de travail. Les travaux menés par PEsola (2008) sur données finlandaises conduisent aux conclusions suivantes qui diffèrent selon le secteur d'activité: un an après la fusion-acquisition, dans le secteur des services, les fusions-acquisitions qui conduisent à une prise de contrôle d'une entreprise par une firme étrangère n'ont pas d'impact significatif sur la probabilité pour un salarié de quitter l'entreprise (ni les acquisitions par des entreprises nationales) par rapport aux salariés des entreprises ne participant pas aux fusions-acquisitions. En revanche, dans l'industrie, les salariés ont de plus fortes chances de quitter l'entreprise juste après une fusion-acquisition, et ce d'autant plus s'il y a prise de contrôle étranger: pour un salarié, la probabilité de départ de l'entreprise est alors augmentée de $+20 \%$, contre $+7 \%$ si l'entreprise bénéficiaire de la fusion-acquisition est finlandaise. Ces effets sur le nombre de salariés qui quittent l'entreprise ont lieu à court terme et ne sont pas pérennes: ils sont essentiellement observés juste après la fusionacquisition et deviennent négligeables les années suivantes. Au niveau des entreprises, l'impact des acquisitions étrangères sur l'emploi apparaît négatif seulement dans le secteur industriel en Finlande alors que les fusions-acquisitions «nationales» ont un effet négatif dans tous les secteurs. Et l'impact sur l'emploi au niveau de l'ensemble de l'économie ne serait pas forcément négatif car PESOLA (2008) montre qu'une grande partie de l'augmentation des départs de salariés après la fusion-acquisition s'explique par une transition vers un emploi dans une autre entreprise.

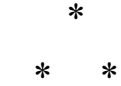

Si on retient les conventions de définition statistique des fusions-acquisitions de la base Citrus de l'Insee, on dénombre sur le territoire français environ 3000 fusions-acquisitions chaque année, sur la période 2000-2004. Elles concernent environ 5000 entreprises par an, cédantes ou bénéficiaires, soit moins de $1 \%$ des entreprises. Les fusions-acquisitions restent donc un phénomène rare. Cependant, comme ces entreprises sont de grande taille, leur poids économique est loin d'être négligeable, en termes d'effectifs notamment. L'évolution de l'emploi des entreprises prenant part 
aux fusions-acquisitions, qui dépend fortement des caractéristiques de la fusion-acquisition, n'est donc pas secondaire pour qui s'intéresse à l'évolution de l'emploi dans l'économie française.

L'exploitation de la base Citrus permet de décrire la répartition des fusions-acquisitions ayant eu lieu sur le territoire français entre 2000 et 2004 en fonction de quatre types de caractéristiques des fusions-acquisitions (repérables dans nos données) qui ressortent dans la littérature économique empirique comme les plus déterminantes pour les conséquences sur l'emploi (le nombre total d'emplois de l'ensemble des entreprises participant à la fusionacquisition ainsi que le devenir sur le marché du travail des salariés de ces entreprises).

1) Concernant le type de fusion-acquisition, les cas de simple changement de propriétaire et d'absorption, où le transfert d'actif est souvent accompagné d'un transfert d'effectif, semblent plus favorables à l'emploi que les scissions partielles et les transferts partiels d'actifs. Les deux premières formes de fusion-acquisition représentent en moyenne un peu moins de la moitié des fusions-acquisitions observées en France au début des années 2000.

2) Le rapport de «dominance» en taille, si on considère les tailles respectives de l'entreprise cédante et de l'entreprise bénéficiaire avant la fusion-acquisition, exercé par la bénéficiaire sur la cédante conduirait à une augmentation du nombre de départs de salariés chez l'entreprise cédante (SMEETs et al.). Il ressort que le déséquilibre est la règle en France. Dans plus de $50 \%$ des cas la bénéficiaire est plus grande que la cédante et dans moins de $10 \%$ elle est de taille comparable.

3) En France, au début des années 2000, plus des deux tiers des fusions-acquisitions ont lieu entre entreprises appartenant à un même secteur d'activité. Du fait du risque d'apparition de doublons, ces fusions-acquisitions intrasectorielles auraient des conséquences moins positives sur l'emploi que les opérations intersectorielles. Cependant la proximité des activités exercées par les entreprises en jeu présente l'avantage de cultures d'entreprise souvent plus proches, ce qui conduirait à réduire le désavantage des salariés issus de la cédante en cas de licenciements (SMEETs et al.).

4) Les fusions-acquisitions contribuent à étendre le poids des groupes de sociétés dans l'économie, les entreprises bénéficiaires des fusions-acquisitions appartenant plus souvent à un groupe que les entreprises cédantes. Seulement $13 \%$ des fusions-acquisitions ont lieu entre entreprises indépendantes (cas de figure le plus favorable à l'emploi). Près de la moitié concerne des entreprises d'un même groupe, situation dont les conséquences sur l'emploi sont moins négatives que dans les autres cas de fusionsacquisitions où des entreprises de groupe interviennent. Les acquisitions d'entreprises par des groupes étrangers, qui auraient un impact négatif plus marqué sur l'emploi (Pesola, 2008) pèsent pour moins de $10 \%$ des transactions en France sur la période 2000-2004.

Ces éléments descriptifs quantitatifs sur les caractéristiques des fusions-acquisitions, les effets théoriquement attendus sur l'emploi des entreprises bénéficiaires et cédantes et les conséquences effectivement observées sur ces entreprises sont de nature à contribuer à guider la mise en œuvre de politiques d'anticipation et de gestion de l'impact des fusionsacquisitions sur l'emploi. Il est important de garder à l'esprit que les conséquences effectivement observées sur l'emploi proviennent à la fois de la stratégie de gestion de la main-d'œuvre décidée au moment de la fusion-acquisition mais aussi, plus indirectement et plus tardivement, du succès ou de l'échec de la fusion-acquisition. 


\section{Bibliographie}

Baldwin J., GoRecki P. (1990), "Mergers and the Competitive Process", Working Paper 773, Queen's University, Department of Economics.

Beauvois M., Rivière P., Beaudeau M., Corbel P. (1999), «CITRUS, système général de traitement des restructurations », document interne, Insee.

Brown C., Medoff J. L. (1988), “The Impact of Firm Acquisition on Labor", in Corporate Takeovers: Causes and Consequences, Alan Auerbach (ed.), University of Chicago Press, 1988, pp. 9-25.

Bunel M., Duhautois R., Gonzalez L. (2008), «Conséquences des fusions-acquisitions sur la gestion de la main-d'œuvre, une analyse empirique sur données françaises pour la vague de la fin des années 1990 », Document d'études, Dares, $\mathrm{n}^{\circ} 133$, janvier 2008.

Conyon, M.J., Girma, S., Thompson, S., Wright, P. (2002), "The impact of mergers and acquisitions on company employment in the United Kingdom", European Economic Review 46, pp. 31-49.

Coutinet N., Sagot-Duvauroux D. (2003), Économie des fusions et acquisitions, collection «Repères », $\mathrm{n}^{\circ} 362$, Paris, La Découverte.
Gugler K., Yurtoglu B.B. (2004), “The effects of mergers on company employment in the USA and Europe", International Journal of Industrial Organization, 22, pp. 481-502.

Margolis D. (2006), "Should Employment Authority Worry about Mergers and Acquisitions?", Portuguese Economic Journal, Vol. 5, No 2.

Pesola H. (2008), "Labour Market Transitions Following Foreign Acquisitions", mimeo.

Ravaran A., (2006), «Négocier les restructurations après la loi du 18 janvier 2005: aspects pratiques», Droit social, 3, p. 298-202.

Sachs-Durand C. (sous la direction de) (2004), La place des salariés dans les restructurations en Europe communautaire, collections de l'université Robert-Schuman, Presses universitaires de Strasbourg.

Smeets V., Ierulli K., Gibbs M. (2008), "Mergers of Equals and Inequals", mimeo. 